\title{
Portfolio Optimization in a Regime-Switching Market with Derivatives
}

\author{
J. Fu, J. Wei†and H. Yang
}

\begin{abstract}
We consider the optimal asset allocation problem in a continuous-time regime-switching market. The problem is to maximize the expected utility of the terminal wealth of a portfolio that contains an option, an underlying stock and a risk-free bond. The difficulty that arises in our setting is finding a way to represent the return of the option by the returns of the stock and the risk-free bond in an incomplete regime-switching market. To overcome this difficulty, we introduce a functional operator to generate a sequence of value functions, and then show that the optimal value function is the limit of this sequence. The explicit form of each function in the sequence can be obtained by solving an auxiliary portfolio optimization problem in a single-regime market. And then the original optimal value function can be approximated by taking the limit. Additionally, we can also show that the optimal value function is a solution to a dynamic programming equation, which leads to the explicit forms for the optimal value function and the optimal portfolio process. Furthermore, we demonstrate that, as long as the current state of the Markov chain is given, it is still optimal for an investor in a multiple-regime market to simply allocate his/her wealth in the same way as in a single-regime market.
\end{abstract}

Key words: Functional operator, Elasticity approach, Portfolio optimization, Regime switching, Dynamic programming principle

AMS Subject Classifications 93E20

\section{Introduction}

It is well known that the basic idea of option pricing in the Black-Scholes model (see Black and Scholes (1973)) is the construction of a non-arbitrage portfolio, which hedges the option's position by taking a position of the opposite sign in the underlying asset with units equal to

\footnotetext{
${ }^{*}$ Department of Statistics and Actuarial Science, The University of Hong Kong, Pokfulam Road, Hong Kong, China, email: fujunster@yahoo.cn

${ }^{\dagger}$ Department of Applied Finance and Actuarial Studies, Faculty of Business and Economics, Macquarie University, Sydney, NSW 2109, Australia, email: jiaqinwei@gmail.com

‡Department of Statistics and Actuarial Science, The University of Hong Kong, Pokfulam Road, Hong Kong, China, email: hlyang@hku.hk
} 
the option's delta. This so-called delta hedge can render the value of a portfolio first orderinsensitive to the change in the underlying price, but does not optimize the portfolio's asset allocation. Mean-variance portfolio optimization in a discrete-time one-period model was proposed by Markowitz (1952). The portfolio optimization problem was further investigated by Merton (1969) and Merton (1971) in a continuous-time setting, which consider an agent who aims to maximize the conditional expectation of the portfolio's utility of the terminal wealth. In these papers, the portfolio consists of only a stock and a risk-free bond, which is known as the reduced portfolio optimization problem and can be solved using the stochastic dynamic programming approach. The martingale and dual approaches to portfolio selection were introduced by Karatzas et al. (1987) and Cox and Huang (1989, 1991). A review of the developments in this area can be found in Korn (2008), which also considers portfolios with option.

In this paper, we optimize a portfolio that comprises an option, an underlying stock and a risk-free bond, and show that the optimal solution is a mixture of Merton's result and the delta hedging strategy. To solve this optimization problem, we adopt the elasticity approach proposed in Kraft (2003) . The elasticity of a derivative with respect to the underlying asset price is defined as the ratio of the percentage change in derivative value to the percentage change in the underlying asset price. By introducing elasticities of the option and portfolio with respect to the stock price, Kraft (2003) demonstrated that the return of an option can be represented in terms of the returns of the stock and the risk-free bond and that the elasticity of the portfolio can be used as the control variable in this optimization problem. In other words, by updating the elasticity of the portfolio rather than the percentage or the amount of wealth invested in the available assets, the portfolio is optimized, and the optimal portfolio process can be obtained by tracking the optimal elasticity.

Here, to model possible changes in the underlying economics, we employ a regime-switching model for the prices of the underlying assets, i.e., the mean rate of return, volatility of the underlying asset price, and return of the risk-free bond depend on a Markov chain. There is a vast body of literature considering the regime-switching model, including Hamilton (1989), Bekaert and Hodrick (1993) and Gray (1996), to name just a few. In addition, Bollen et al. (2000) considered the regimes in the volatilities of currency option prices. More evidence of the regimes in the returns of stocks or bonds can be found in Garcia and Perron (1996), Ang and Chen (2002) and Guidolin and Timmermann (2007). Considering a regime-switching model, the portfolio optimization problem is studied by Çelikyurt and Özekici (2007) with mean-variance criterion, and by Çanakoğlu and Özekici (2012) with HARA utility functions.

However, the market becomes incomplete under the regime-switching model, which results in difficulties using the elasticity approach, because the option cannot be completely replicated by the stock and risk-free asset in an incomplete market. To overcome these difficulties, we take the following steps. First, we introduce a functional operator and then generate a sequence of value functions by applying this operator repeatedly. Second, we prove that the optimal value function is the limit of this sequence. Third, to obtain the explicit solution for each function in the sequence, we propose an auxiliary optimization problem in a single-regime market in which the elasticity approach is applicable. Finally, the original optimal value function can be approximated by taking the limit. Additionally, the optimal value function can be shown to satisfy a dynamic programming equation, which leads to a PDE. Hence, the exact solution for the optimal value function and the optimal portfolio process are also available. In sum, the original problem in a multiple-regime market can be reduced to an optimization problem with only a single regime. And it shows that, as long as the current state of the Markov chain is 
given, an investor in a multiple-regime market simply needs to allocate his/her wealth in the same way as in a single-regime market to achieve an optimal strategy.

The remainder of the paper is organized as follows. Section 2 formulates the portfolio optimization problem in a regime-switching market. Section 3 introduces the functional operator and derives a number of properties. On the basis of these properties, Section 4 provides an approximate solution by solving an auxiliary optimization problem. Section 5 then gives an exact result by solving the dynamic programming equation, and obtains the optimal portfolio process. Finally, Section 6 concludes the paper.

\section{Optimization in a Regime-Switching Market}

In the following, we denote the matrices and vectors by letters in boldface, and their transpose by a superscript $T r$. Considering a fixed time horizon $[0, T]$, we divide the market mode into $d$ different regimes. We model the market mode by a continuous-time stationary Markov chain $\{\boldsymbol{\alpha}(t)\}$ that is defined on the complete probability space $(\Omega, \mathscr{F}, \mathrm{P})$, where $\mathrm{P}$ denotes physical probability.

Without loss of generality, we can identify the state space of $\{\boldsymbol{\alpha}(t)\}$ as a finite set of unit vectors $\mathbb{E}:=\left(\mathbf{e}_{1}, \mathbf{e}_{2}, \cdots, \mathbf{e}_{d}\right)$, where $\mathbf{e}_{i} \in \mathbb{R}^{d}$ are column vectors with unity in the $i$-th position and zero elsewhere. We call $\mathbb{E}$ the canonical space of the chain $\{\boldsymbol{\alpha}(t)\}$. Denote by $\mathbf{Q}=\left(q_{i j}\right)_{d \times d}$ the generator of the chain $\{\boldsymbol{\alpha}(t)\}$ under P. We then have the following semi-martingale representation for the chain,

$$
\boldsymbol{\alpha}(t)=\boldsymbol{\alpha}(0)+\int_{0}^{t} \mathbf{Q} \boldsymbol{\alpha}(s) d s+\mathbf{M}(t),
$$

where $\mathbf{M}(t)$ is an $\mathbb{R}^{d}$-valued martingale with respect to the filtration generated by $\{\boldsymbol{\alpha}(t)\}$.

In a regime-switching market, the risk-free bond process $\{M(t)\}$ and a risky stock price process $\{S(t)\}$ are assumed to evolve as

$$
\begin{aligned}
\frac{d M(t)}{M(t)} & =\langle\mathbf{r}, \boldsymbol{\alpha}(t)\rangle d t \\
\frac{d S(t)}{S(t)} & =\langle\boldsymbol{\mu}, \boldsymbol{\alpha}(t)\rangle d t+\langle\boldsymbol{\sigma}, \boldsymbol{\alpha}(t)\rangle d B(t),
\end{aligned}
$$

where $\mathbf{r}=\left(r_{1}, r_{2}, \cdots, r_{d}\right)^{T r}, \boldsymbol{\mu}=\left(\mu_{1}, \mu_{2}, \cdots, \mu_{d}\right)^{T r}, \boldsymbol{\sigma}=\left(\sigma_{1}, \sigma_{2}, \cdots, \sigma_{d}\right)^{T r}$ with $\sigma_{i}>0$ for all $i \in \mathbb{D}:=\{1, \ldots, d\}$, and $\{B(t)\}$ is a standard Brownian motion independent of $\{\boldsymbol{\alpha}(t)\}$. Both of these processes are $\left\{\mathscr{F}_{t}\right\}$-adapted with $\left\{\mathscr{F}_{t}\right\}$ satisfying the usual conditions.

For a portfolio comprising an option, an underlying stock and a risk-free bond, let $\{O(t)\}$ be the price process of the option which is written on the stock. Let $\pi_{S}$ and $\pi_{O}$ denote the percentages of wealth invested in the stock and option, respectively, and $\pi(s):=\left(\pi_{S}(s), \pi_{O}(s)\right)$ for all $s \in[t, T]$. Then, the wealth process $\left\{W_{\pi}(t)\right\}$ of the portfolio evolves as

$$
\frac{d W_{\pi}(t)}{W_{\pi}(t)}=\pi_{S}(t) \frac{d S(t)}{S(t)}+\pi_{O}(t) \frac{d O(t)}{O(t)}+\left[1-\pi_{S}(t)-\pi_{O}(t)\right] \frac{d M(t)}{M(t)} .
$$


Here, the subscript of $\pi$ represents the dependence of wealth process on portfolio process $\{\pi(s)\}_{s=t}^{T}$.

At any time $t \leq T$, our objective is to maximize the expected utility of the terminal wealth. Denote by

$$
\Pi_{t}:=\left\{\{\pi(s)\}_{s=t}^{T}\right\}
$$

the set of all admissible strategies over $[t, T]$. Then define

$$
V_{\pi}(t, w, i):=\mathrm{E}_{t, w, i}\left[U\left(W_{\pi}(T)\right)\right], \quad V(t, w, i):=\sup _{\pi \in \Pi_{t}} V_{\pi}(t, w, i),
$$

where $U(\cdot)$ is a utility function with $U^{\prime}>0$ and $U^{\prime \prime}<0$, and

$$
\mathrm{E}_{t, w, i}[\cdot]=\mathrm{E}\left[\cdot \mid W_{\pi}(t)=w, \boldsymbol{\alpha}(t)=\mathbf{e}_{i}\right] .
$$

It is obvious that $V$ satisfies the following boundary condition:

$$
V(T, w, i)=V_{\pi}(T, w, i)=U(w), \quad \forall w \geq 0, i \in \mathbb{D} .
$$

In summary, given a pair of $(t, i) \in[0, T] \times \mathbb{D}$, our optimization problem is to find a strategy $\hat{\pi} \in \Pi_{t}$ such that

$$
V_{\hat{\pi}}(t, w, i)=V(t, w, i), \quad \forall w \in[0,+\infty[,
$$

and to obtain function $V(t, w, i)$ for each $(t, w, i) \in[0, T] \times[0,+\infty[\times \mathbb{D}$.

\section{Functional Operator}

In this section, after introducing a functional operator, we show that the aforementioned optimal value function can be obtained by applying this operator repeatedly.

In what follows, unless otherwise specified, a vector function in the form of

$$
\mathbf{v}(t, w)=(v(t, w, 1), v(t, w, 2), \cdots, v(t, w, d)),
$$

means that $v(t, w, i):=v\left(t, w, \mathbf{e}_{i}\right)$ for all $i \in \mathbb{D}$, and, when we use $\leq$ or $\geq$ for two vectors, it means that the relation holds for each element.

For a test function $\mathbf{v}$, we introduce a functional operator denoted by $\mathscr{M}$ :

$$
\begin{aligned}
\mathscr{M} v(t, w, i) & :=\sup _{\pi \in \Pi_{t}} \mathrm{E}_{t, w, i}\left[v\left(\xi_{t, 1} \wedge T, W_{\pi}\left(\xi_{t, 1} \wedge T\right), \boldsymbol{\alpha}\left(\xi_{t, 1} \wedge T\right)\right)\right] \\
& =\sup _{\pi \in \Pi_{t}} \mathrm{E}_{t, w, i}\left[1_{\left\{\xi_{t, 1}<T\right\}} v\left(\xi_{t, 1}, W_{\pi}\left(\xi_{t, 1}\right), \boldsymbol{\alpha}\left(\xi_{t, 1}\right)\right)+1_{\left\{\xi_{t, 1} \geq T\right\}} U\left(W_{\pi}(T)\right)\right],
\end{aligned}
$$

where $\xi_{t, n}$ denotes the $n$-th jump time of Markov chain $\{\boldsymbol{\alpha}(s)\}_{s=t}^{\infty}$ for $n \in \mathbb{N}$ with $\xi_{t, 0}=t$, and the use of $\xi_{t, 1}$ indicates that the right-hand side of (1) is an optimization problem in a single-regime world. We also write

$$
\mathscr{M} \mathbf{v}(t, w):=(\mathscr{M} v(t, w, 1), \mathscr{M} v(t, w, 2), \cdots, \mathscr{M} v(t, w, d)) .
$$

This functional operator $\mathscr{M}$ has the following properties. 
Lemma 3.1 If $\mathbf{v}_{1}(t, w) \geq \mathbf{v}_{2}(t, w), \forall(t, w) \in[0, T] \times[0,+\infty[$, then

$$
\mathscr{M} \mathbf{v}_{1}(t, w) \geq \mathscr{M} \mathbf{v}_{2}(t, w), \quad \forall(t, w) \in[0, T] \times[0, \infty[.
$$

Lemma 3.2 For any $(t, w, i) \in[0, T] \times[0, \infty[\times \mathbb{D}$, define

$$
H_{0}(t, w, i):=\mathrm{E}_{t, w, i}\left[U\left(w \cdot \exp \left(\int_{t}^{T}\langle\mathbf{r}, \boldsymbol{\alpha}(s)\rangle d s\right)\right)\right],
$$

which is the expected utility of the terminal wealth if we allocate all of the wealth in the risk-free bond over $[t, T]$, and

$$
H_{n+1}(t, w, i):=\mathscr{M} H_{n}(t, w, i), \quad \text { for } n \in \mathbb{N} .
$$

Then, for any $(t, i) \in[0, T] \times \mathbb{D},\left\{H_{n}(t, \cdot, i)\right\}_{n \in \mathbb{N}}$ is an increasing sequence of functions on $[0, \infty[$.

Proof: Since

$$
H_{1}(t, w, i)=\mathscr{M} H_{0}(t, w, i), \quad \forall(t, w, i) \in[0, T] \times[0, \infty[\times \mathbb{D},
$$

we have

$$
\begin{aligned}
H_{1}(t, w, i)= & \sup _{\pi \in \Pi_{t}} \mathrm{E}_{t, w, i}\left\{1_{\left\{\xi_{t, 1}<T\right\}} H_{0}\left(\xi_{t, 1}, W_{\pi}\left(\xi_{t, 1}\right), \boldsymbol{\alpha}\left(\xi_{t, 1}\right)\right)+1_{\left\{\xi_{t, 1} \geq T\right\}} U\left(W_{\pi}(T)\right)\right\} \\
= & \sup _{\pi \in \Pi_{t}} \mathrm{E}_{t, w, i}\left\{1_{\left\{\xi_{t, 1}<T\right\}} \mathrm{E}_{\xi_{t, 1}, W_{\pi}\left(\xi_{t, 1}\right), \boldsymbol{\alpha}\left(\xi_{t, 1}\right)}\left[U\left(W_{\pi}\left(\xi_{t, 1}\right) \cdot \exp \left(\int_{\xi_{t, 1}}^{T}\langle\mathbf{r}, \boldsymbol{\alpha}(s)\rangle d s\right)\right)\right]\right. \\
& \left.+1_{\left\{\xi_{t, 1} \geq T\right\}} U\left(W_{\pi}(T)\right)\right\} \\
= & \sup _{\pi \in \Pi_{t}} \mathrm{E}_{t, w, i}\left\{1_{\left\{\xi_{t, 1}<T\right\}} U\left[W_{\pi}\left(\xi_{t, 1}\right) \cdot \exp \left(\int_{\xi_{t, 1}}^{T}\langle\mathbf{r}, \boldsymbol{\alpha}(s)\rangle d s\right)\right]+1_{\left\{\xi_{t, 1} \geq T\right\}} U\left(W_{\pi}(T)\right)\right\} \\
\geq & \mathrm{E}_{t, w, i}\left\{U\left[w \cdot \exp \left(\int_{t}^{T}\langle\mathbf{r}, \boldsymbol{\alpha}(s)\rangle d s\right)\right]\right\} \\
= & H_{0}(t, w, i),
\end{aligned}
$$

where $\mathrm{E}_{.,, \mathbf{e}_{i}}=\mathrm{E}_{.,, i}$. The result then follows from Lemma 3.1.

Lemma 3.3 For any $(t, w, i) \in[0, T] \times[0, \infty[\times \mathbb{D}$, let

$$
V_{n}(t, w, i):=\sup _{\pi \in \Pi_{t, n}} V_{\pi}(t, w, i)
$$

where

$$
\Pi_{t, n}:=\left\{\{\pi(s)\}_{s=t}^{T}: \pi_{S}(s) \equiv \pi_{O}(s) \equiv 0, \forall s \geq \xi_{t, n}\right\}, \quad \text { for } n \in \mathbb{N},
$$

which is the set of strategies over $[t, T]$ allocating all of the wealth in the risk-free bond after the n-th jump of the Markov chain $\{\boldsymbol{\alpha}(s)\}_{s=t}^{T}$. Then, for any $(t, w) \in[0, T] \times[0, \infty[$, we have

$$
\mathbf{V}_{n}(t, w)=\mathbf{H}_{n}(t, w), \quad \forall n \in \mathbb{N} .
$$


Proof: We can prove the result through mathematical induction. First, by the definition of $\mathbf{H}_{0}$, it is obvious that

$$
\mathbf{V}_{0}(t, w)=\mathbf{H}_{0}(t, w), \quad \forall(t, w) \in[0, T] \times[0, \infty[.
$$

Second, based on the assumption that

$$
\mathbf{V}_{n}(t, w)=\mathbf{H}_{n}(t, w), \quad \forall(t, w) \in[0, T] \times[0, \infty[
$$

we prove that the equality also holds for $n+1$ as follows.

To show that $V_{n+1}(t, w, i) \leq H_{n+1}(t, w, i)$, it suffices to show that

$$
V_{n+1}(t, w, i)-\varepsilon \leq H_{n+1}(t, w, i), \quad \forall \varepsilon>0 .
$$

Indeed, for an arbitrarily given $\varepsilon>0$, we can find a strategy $\pi \in \Pi_{t, n+1}$ over $[t, T]$, such that

$$
V_{\pi}(t, w, i) \geq V_{n+1}(t, w, i)-\varepsilon,
$$

and, by defining a strategy over $\left[\xi_{t, 1}, T\right]$ as

$$
\pi^{*}(s):=\pi(s), \quad \text { for } s \in\left[\xi_{t, 1}, T\right],
$$

we can obtain $\pi^{*} \in \Pi_{\xi_{t, 1}, n}$ and

$$
\begin{aligned}
V_{\pi}(t, w, i) & =\mathrm{E}_{t, w, i}\left[U\left(W_{\pi}(T)\right)\right] \\
& =\mathrm{E}_{t, w, i}\left[1_{\left\{\xi_{t, 1}<T\right\}} V_{\pi^{*}}\left(\xi_{t, 1}, W_{\pi}\left(\xi_{t, 1}\right), \boldsymbol{\alpha}\left(\xi_{t, 1}\right)\right)+1_{\left\{\xi_{t, 1} \geq T\right\}} U\left(W_{\pi}(T)\right)\right] \\
& \leq \mathrm{E}_{t, w, i}\left[1_{\left\{\xi_{t, 1}<T\right\}} V_{n}\left(\xi_{t, 1}, W_{\pi}\left(\xi_{t, 1}\right), \boldsymbol{\alpha}\left(\xi_{t, 1}\right)\right)+1_{\left\{\xi_{t, 1} \geq T\right\}} U\left(W_{\pi}(T)\right)\right] \\
& =\mathrm{E}_{t, w, i}\left[1_{\left\{\xi_{t, 1}<T\right\}} H_{n}\left(\xi_{t, 1}, W_{\pi}\left(\xi_{t, 1}\right), \boldsymbol{\alpha}\left(\xi_{t, 1}\right)\right)+1_{\left\{\xi_{t, 1} \geq T\right\}} U\left(W_{\pi}(T)\right)\right] \\
& \leq H_{n+1}(t, w, i),
\end{aligned}
$$

which, combined with (5), implies (4).

To show that $V_{n+1}(t, w, i) \geq H_{n+1}(t, w, i)$, we need only show that

$$
V_{n+1}(t, w, i)+2 \varepsilon \geq H_{n+1}(t, w, i), \quad \forall \varepsilon>0 .
$$

Indeed, for an arbitrarily given $\varepsilon>0$, we can find a strategy $\pi \in \Pi_{t}$, such that

$$
H_{n+1}(t, w, i) \leq \mathrm{E}_{t, w, i}\left[1_{\left\{\xi_{t, 1}<T\right\}} H_{n}\left(\xi_{t, 1}, W_{\pi}\left(\xi_{t, 1}\right), \boldsymbol{\alpha}\left(\xi_{t, 1}\right)\right)+1_{\left\{\xi_{t, 1} \geq T\right\}} U\left(W_{\pi}(T)\right)\right]+\varepsilon .
$$

In addition, for this $\varepsilon$ we can also find another strategy $\pi^{\prime} \in \Pi_{\xi_{t, 1}, n}$ over $\left[\xi_{t, 1}, T\right]$, such that

$$
V_{n}\left(\xi_{t, 1}, W_{\pi}\left(\xi_{t, 1}\right), \boldsymbol{\alpha}\left(\xi_{t, 1}\right)\right) \leq V_{\pi^{\prime}}\left(\xi_{t, 1}, W_{\pi}\left(\xi_{t, 1}\right), \boldsymbol{\alpha}\left(\xi_{t, 1}\right)\right)+\varepsilon
$$

Then, by defining a strategy $\tilde{\pi} \in \Pi_{t, n+1}$ over $[t, T]$ as

$$
\tilde{\pi}(s)= \begin{cases}\pi(s), & t \leq s<\xi_{t, 1} \wedge T \\ \pi^{\prime}(s), & \xi_{t, 1} \wedge T \leq s \leq T\end{cases}
$$


it follows from (7) and (8) that

$$
\begin{aligned}
H_{n+1}(t, w, i) & \leq \mathrm{E}_{t, w, i}\left[1_{\left\{\xi_{t, 1}<T\right\}} V_{\pi^{\prime}}\left(\xi_{t, 1}, W_{\pi}\left(\xi_{t, 1}\right), \boldsymbol{\alpha}\left(\xi_{t, 1}\right)\right)+1_{\left\{\xi_{t, 1} \geq T\right\}} U\left(W_{\pi}(T)\right)\right]+2 \varepsilon \\
& =V_{\tilde{\pi}}(t, w, i)+2 \varepsilon \\
& \leq V_{n+1}(t, w, i)+2 \varepsilon,
\end{aligned}
$$

which is the same as (6).

Proposition 3.1 For any $(t, w, i) \in[0, T] \times[0, \infty[\times \mathbb{D}$, we have

$$
\lim _{n \rightarrow \infty} H_{n}(t, w, i)=V(t, w, i) .
$$

Proof: For any $(t, w, i) \in[0, T] \times\left[0, \infty\left[\times \mathbb{D}\right.\right.$, owing to Lemma 3.2, $\lim _{n \rightarrow \infty} H_{n}(t, w, i)$ is well defined, and it follows from Lemma 3.3 that

$$
H(t, w, i):=\lim _{n \rightarrow \infty} H_{n}(t, w, i)=\lim _{n \rightarrow \infty} V_{n}(t, w, i) \leq V(t, w, i) .
$$

To prove the opposite inequality, it suffices to show that, for any $\pi \in \Pi_{t}$,

$$
H(t, w, i) \geq V_{\pi}(t, w, i), \quad \forall(t, w, i) \in[0, T] \times[0, \infty[\times \mathbb{D} .
$$

Indeed, for an arbitrarily given strategy $\pi \in \Pi_{t}$, we can define a strategy $\pi_{n} \in \Pi_{t, n}$ over $[t, T]$ as

$$
\pi_{n}(s)= \begin{cases}\pi(s), & t \leq s<\xi_{t, n} \wedge T \\ 0, & \xi_{t, n} \wedge T \leq s \leq T\end{cases}
$$

and we then have

$$
\begin{aligned}
& V_{\pi}(t, w, i)-V_{\pi_{n}}(t, w, i) \\
= & \mathrm{E}_{t, w, i}\left\{1_{\left\{\xi_{t, 1}<T\right\}}\left[V_{\pi}\left(\xi_{t, 1}, W_{\pi}\left(\xi_{t, 1}\right), \boldsymbol{\alpha}\left(\xi_{t, 1}\right)\right)-V_{\pi_{n}}\left(\xi_{t, 1}, W_{\pi}\left(\xi_{t, 1}\right), \boldsymbol{\alpha}\left(\xi_{t, 1}\right)\right)\right]\right\} \\
= & \mathrm{E}_{t, w, i}\left\{1_{\left\{\xi_{t, 1}<\xi_{t, n}<T\right\}}\left[V_{\pi}\left(\xi_{t, 1}, W_{\pi}\left(\xi_{t, 1}\right), \boldsymbol{\alpha}\left(\xi_{t, 1}\right)\right)-V_{\pi_{n}}\left(\xi_{t, 1}, W_{\pi}\left(\xi_{t, 1}\right), \boldsymbol{\alpha}\left(\xi_{t, 1}\right)\right)\right]\right\} \\
\rightarrow & 0,
\end{aligned}
$$

as $n \rightarrow \infty$. Hence, for any $\varepsilon>0$, there exists an $n(\varepsilon) \in \mathbb{N}$ such that

$$
H(t, w, i) \geq H_{n(\varepsilon)}(t, w, i)=V_{n(\varepsilon)}(t, w, i) \geq V_{\pi_{n(\varepsilon)}}(t, w, i) \geq V_{\pi}(t, w, i)-\varepsilon,
$$

which implies (9).

From this proposition, the optimal value function can also be shown to be the fixed point of the functional operator of $\mathscr{M}$.

Proposition 3.2 The optimal value function $\mathbf{V}$ is the smallest non-negative solution of the dynamic programming equation $\mathbf{V}=\mathscr{M} \mathbf{V}$, i.e., for any $(t, w, i) \in[0, T] \times[0, \infty[\times \mathbb{D}, \mathbf{V}$ satisfies

$$
V(t, w, i)=\sup _{\pi \in \Pi_{t}} \mathrm{E}_{t, w, i}\left[1_{\left\{\xi_{t, 1}<T\right\}} V\left(\xi_{t, 1}, W_{\pi}\left(\xi_{t, 1}\right), \boldsymbol{\alpha}\left(\xi_{t, 1}\right)\right)+1_{\left\{\xi_{t, 1} \geq T\right\}} U\left(W_{\pi}(T)\right)\right] .
$$


Proof: From Proposition 3.1, it is obvious that $\mathbf{V}=\mathscr{M} \mathbf{V}$. Suppose that there is another fixed point of $\mathscr{M}$ denoted by $\tilde{\mathbf{V}}$. Then, by the definition of $\mathbf{H}_{n}$ for any $n \in \mathbb{N}$, for any $(t, w, i) \in$ $[0, T] \times\left[0, \infty\left[\times \mathbb{D}\right.\right.$, we have $\tilde{V}(t, w, i) \geq H_{0}(t, w, i)$, and

$$
\tilde{V}(t, w, i)=\mathscr{M} \tilde{V}(t, w, i) \geq \mathscr{M} H_{0}(t, w, i)=H_{1}(t, w, i),
$$

which further implies that $\tilde{V}(t, w, i) \geq H_{n}(t, w, i)$ for any $n \in \mathbb{N}$, and

$$
\tilde{V}(t, w, i) \geq V(t, w, i), \quad \forall(t, w, i) \in[0, T] \times[0, \infty[\times \mathbb{D} .
$$

The proof is completed.

In Proposition 3.1, we conclude that the optimal value function $\mathbf{V}$ can be obtained by the limit of a sequence of functions, which leads to an auxiliary optimization problem. An approximate solution for $\mathbf{V}$ is given in the next section. Proposition 3.2, however, shows that $\mathbf{V}$ is also the solution to a dynamic programming equation, which is considered in Section 5.

\section{Approximate Solution via an Auxiliary Problem}

\subsection{Auxiliary Problem}

By Proposition 3.1, optimal value function $\mathbf{V}$ can be obtained by iteration (3). Then, we need only consider, for an given function $\mathbf{v}$, how to obtain the explicit form of $\mathscr{M} \mathbf{v}$. Note that we can simplify the right-hand side of (1) as follows.

$$
\begin{aligned}
\mathscr{M} v(t, w, i) & =\sup _{\pi \in \Pi_{t}} \mathrm{E}_{t, w, i}\left[1_{\left\{\xi_{t, 1}<T\right\}} v\left(\xi_{t, 1}, W_{\pi}\left(\xi_{t, 1}\right), \boldsymbol{\alpha}\left(\xi_{t, 1}\right)\right)+1_{\left\{\xi_{t, 1} \geq T\right\}} U\left(W_{\pi}(T)\right)\right] \\
& =\sup _{\pi \in \Pi_{t}} \mathrm{E}_{t, w, i}\left[\int_{t}^{T} e^{-q_{i}(s-t)} \sum_{j \neq i} q_{i j} v\left(s, W_{\pi}^{(i)}(s), j\right) d s+e^{-q_{i}(T-t)} U\left(W_{\pi}^{(i)}(T)\right)\right] \\
& =e^{q_{i} t} \sup _{\pi \in \Pi_{t}} \mathrm{E}_{t, w, i}\left[\int_{t}^{T} e^{-q_{i} s} \sum_{j \neq i} q_{i j} v\left(s, W_{\pi}^{(i)}(s), j\right) d s+e^{-q_{i} T} U\left(W_{\pi}^{(i)}(T)\right)\right]
\end{aligned}
$$

where

$$
q_{i}:=\sum_{j \neq i} q_{i j}
$$

and $W_{\pi}^{(i)}$ denotes the wealth process driven by $\pi$ in the single-regime world $i$, which is given by (18). We have thus obtained an auxiliary dynamic programming problem, which, for each fixed $i \in \mathbb{D}$, is an optimization problem in a single-regime world.

\subsection{Elasticity Approach in a Single-Regime World}

To solve this auxiliary problem, we derive the dynamics of $\left\{W_{\pi}^{(i)}(t)\right\}$ using the elasticity approach. In single-regime world $i$, let $S^{(i)}(t)$ and $M^{(i)}(t)$ denote the prices of the stock and 
risk-free bond, respectively. Then,

$$
\begin{aligned}
\frac{d S^{(i)}(t)}{S^{(i)}(t)} & =\mu_{i} d t+\sigma_{i} d B(t), \\
\frac{d M^{(i)}(t)}{M^{(i)}(t)} & =r_{i} d t,
\end{aligned}
$$

and we can obtain risk-neutral probability $\mathbb{Q}_{i}$, under which

$$
\frac{d S^{(i)}(t)}{S^{(i)}(t)}=r_{i} d t+\sigma_{i} d B^{\mathbb{Q}_{i}}(t),
$$

where $B^{\mathbb{Q}_{i}}$ is a standard Brownian motion under $\mathbb{Q}_{i}$. Let $O^{(i)}\left(t, S^{(i)}(t)\right)$ denote the value of a given contingent claim traded on the stock at time $t$. Application of Itô's formula to its discounted value, which is a martingale under $\mathbb{Q}_{i}$, yields

$$
\frac{\partial O^{(i)}\left(t, S^{(i)}(t)\right)}{\partial t}+r_{i} S^{(i)}(t) \frac{\partial O^{(i)}\left(t, S^{(i)}(t)\right)}{\partial S}+\frac{1}{2} \sigma_{i}^{2}\left(S^{(i)}(t)\right)^{2} \frac{\partial^{2} O^{(i)}\left(t, S^{(i)}(t)\right)}{\partial S^{2}}=r_{i} O^{(i)}\left(t, S^{(i)}(t)\right) .
$$

Next, for a delta-neutral portfolio that takes the form

$$
X^{(i)}(t)=-O^{(i)}\left(t, S^{(i)}(t)\right)+\frac{\partial O^{(i)}\left(t, S^{(i)}(t)\right)}{\partial S} \cdot S^{(i)}(t),
$$

from the self-financing property, we have

$$
\begin{aligned}
d X^{(i)}(t) & =-d O^{(i)}\left(t, S^{(i)}(t)\right)+\frac{\partial O^{(i)}\left(t, S^{(i)}(t)\right)}{\partial S} d S^{(i)}(t) \\
& =-\frac{\partial O^{(i)}\left(t, S^{(i)}(t)\right)}{\partial t} d t-\frac{1}{2} \sigma_{i}^{2}\left(S^{(i)}(t)\right)^{2} \frac{\partial^{2} O^{(i)}\left(t, S^{(i)}(t)\right)}{\partial S^{2}} d t \\
& =r_{i}\left[S^{(i)}(t) \frac{\partial O^{(i)}\left(t, S^{(i)}(t)\right)}{\partial S}-O^{(i)}\left(t, S^{(i)}(t)\right)\right] d t
\end{aligned}
$$

Rearrangement of this equation then implies that

$$
\begin{aligned}
d O^{(i)}\left(t, S^{(i)}(t)\right) & =-r_{i}\left[S^{(i)}(t) \frac{\partial O^{(i)}\left(t, S^{(i)}(t)\right)}{\partial S}-O^{(i)}\left(t, S^{(i)}(t)\right)\right] d t+\frac{\partial O^{(i)}\left(t, S^{(i)}(t)\right)}{\partial S} d S^{(i)}(t) \\
& =\left[r_{i} O^{(i)}\left(t, S^{(i)}(t)\right)+\frac{\partial O^{(i)}\left(t, S^{(i)}(t)\right)}{\partial S}\left(\mu_{i}-r_{i}\right) S^{(i)}(t)\right] d t+\sigma_{i} S^{(i)}(t) \frac{\partial O^{(i)}\left(t, S^{(i)}(t)\right)}{\partial S} d B(t),
\end{aligned}
$$

which further leads to

$$
\frac{d O^{(i)}\left(t, S^{(i)}(t)\right)}{O^{(i)}\left(t, S^{(i)}(t)\right)}=\left[r_{i}+\varepsilon_{O}^{(i)}\left(\mu_{i}-r_{i}\right)\right] d t+\varepsilon_{O}^{(i)} \sigma_{i} d B(t),
$$

where we introduce the elasticity of the option price with respect to the stock price defined as

$$
\varepsilon_{O}^{(i)}:=\frac{\partial O^{(i)}\left(t, S^{(i)}(t)\right)}{\partial S} \cdot \frac{S^{(i)}(t)}{O^{(i)}\left(t, S^{(i)}(t)\right)} .
$$


Recall that the wealth process $\left\{W_{\pi}^{(i)}(t)\right\}$ of a portfolio comprising an option, an underlying stock and a risk-free bond evolves as

$$
\frac{d W_{\pi}^{(i)}(t)}{W_{\pi}^{(i)}(t)}=\pi_{S} \frac{d S^{(i)}(t)}{S^{(i)}(t)}+\pi_{O} \frac{d O^{(i)}\left(t, S^{(i)}(t)\right)}{O^{(i)}\left(t, S^{(i)}(t)\right)}+\left(1-\pi_{S}-\pi_{O}\right) \frac{d M^{(i)}(t)}{M^{(i)}(t)}
$$

Substituting (12), (13) and (17) leads to

$$
\begin{aligned}
\frac{d W_{\varepsilon^{(i)}}^{(i)}(t)}{W_{\varepsilon^{(i)}}^{(i)}(t)} & =\left[\pi_{O}\left(r_{i}+\varepsilon_{O}^{(i)}\left(\mu_{i}-r_{i}\right)\right)+\pi_{S} \mu_{i}+\left(1-\pi_{S}-\pi_{O}\right) r_{i}\right] d t+\left[\pi_{O} \varepsilon_{O}^{(i)}+\pi_{S}\right] \sigma_{i} d B(t) \\
& =\left[\pi_{O} \varepsilon_{O}^{(i)}\left(\mu_{i}-r_{i}\right)+\pi_{S}\left(\mu_{i}-r_{i}\right)+r_{i}\right] d t+\left[\pi_{O} \varepsilon_{O}^{(i)}+\pi_{S}\right] \sigma_{i} d B(t) \\
& =\left[\varepsilon^{(i)}\left(\mu_{i}-r_{i}\right)+r_{i}\right] d t+\varepsilon^{(i)} \sigma_{i} d B(t)
\end{aligned}
$$

where $\varepsilon^{(i)}$ denotes the elasticity of the entire portfolio with respect to the stock price, i.e.,

$$
\varepsilon^{(i)}:=\pi_{O} \varepsilon_{O}^{(i)}+\pi_{S}
$$

Since the $\varepsilon^{(i)}$ is the single control variable for the dynamics of the wealth process in the singleregime world $i$, we replaced the subscript of $\pi$ of the wealth process by the new control variable $\varepsilon^{(i)}$. For this single-regime optimization problem, we first determine the optimal elasticity, which in a complete market is independent of a specific asset. Then a strategy is computed which tracks this optimal elasticity (see Subsection 5.3).

\subsection{Solution to the Auxiliary Problem}

For auxiliary optimization problem (11), because $\varepsilon^{(i)}$ is a single control variable, as derived in the foregoing section, we rewrite (11) and denote

$$
\begin{aligned}
M(t, w, i): & =\mathscr{M} v(t, w, i) \\
& =e^{q_{i} t} \cdot \sup _{\varepsilon^{(i)}} \mathrm{E}_{t, w, i}\left[\int_{t}^{T} e^{-q_{i} s} \sum_{j \neq i} q_{i j} v\left(s, W_{\varepsilon^{(i)}}^{(i)}(s), j\right) d s+e^{-q_{i} T} U\left(W_{\varepsilon^{(i)}}^{(i)}(T)\right)\right] .
\end{aligned}
$$

We then obtain a PDE for $M(\cdot, \cdot, i)$ on $[0, T[\times] 0,+\infty[$.

Proposition 4.1 For each $i \in \mathbb{D}$, the function of $M(\cdot, \cdot, i)$ satisfies

$$
\begin{aligned}
& \sigma_{i}^{2}\left[M_{t}(t, w, i)+r_{i} w M_{W}(t, w, i)-q_{i} M(t, w, i)+\sum_{j \neq i} q_{i j} v(t, w, j)\right] M_{W W}(t, w, i) \\
= & \frac{1}{2}\left(\mu_{i}-r_{i}\right)^{2} M_{W}^{2}(t, w, i)
\end{aligned}
$$

on $[0, T[\times] 0,+\infty[$ and has boundary condition

$$
M(T, w, i)=U(w), \quad w \in[0,+\infty[.
$$


Proof: For each fixed $i \in \mathbb{D}$, considering (20) and the dynamics of $\left\{W_{\varepsilon^{(i)}}^{(i)}(t)\right\}$ given by (19), we have the following Hamilton-Jacobi-Bellman equation.

$$
\begin{aligned}
0= & \sup _{\varepsilon^{(i)}}\left\{M_{t}(t, w, i)-q_{i} M(t, w, i)+\left[\varepsilon^{(i)}\left(\mu_{i}-r_{i}\right)+r_{i}\right] w M_{W}(t, w, i)\right. \\
& \left.+\frac{1}{2} \sigma_{i}^{2}\left(\varepsilon^{(i)}\right)^{2} w^{2} M_{W W}(t, w, i)+\sum_{j \neq i} q_{i j} v(t, w, j)\right\}
\end{aligned}
$$

on $[0, T[\times] 0,+\infty[$, with its first-order condition leading to the optimal elasticity

$$
\hat{\varepsilon}^{(i)}=\hat{\pi}_{O} \varepsilon_{O}^{(i)}+\hat{\pi}_{S}=-\frac{\left(\mu_{i}-r_{i}\right) M_{W}(t, w, i)}{\sigma_{i}^{2} w M_{W W}(t, w, i)} .
$$

By substitution, we can then obtain PDE (21). The boundary condition follows immediately from (20). Furthermore, it follows from the standard verification theorem (see, e.g. Fleming and Soner (2006)) that the solution to the PDE (21) is indeed the function $M(t, w, i)$.

Although solving this PDE for $\mathbf{M}$ appears difficult, we know that $\mathbf{M}$ is the result of one-step iteration (3), and the first step of this iteration involves finding $\mathscr{M} \mathbf{H}_{0}$, with $\mathbf{H}_{0}$ given by (2). Accordingly, we first attempt to solve the PDE with $\mathbf{v}$ replaced by $\mathbf{H}_{0}$, and then obtain a general explicit form of $\mathbf{H}_{n}$. In addition, we restrict ourselves to the constant relative risk aversion (CRRA) utility, i.e.,

$$
U(x)= \begin{cases}\frac{x^{\gamma}}{\gamma}, & \gamma<1, \gamma \neq 0 \\ \ln x, & \gamma=0\end{cases}
$$

for $x>0$. We then obtain the following result.

Proposition 4.2 If the agent has a CRRA utility of

$$
U(x)=\frac{x^{\gamma}}{\gamma}, \quad \text { for } x \geq 0,
$$

where $\gamma<1$ and $\gamma \neq 0$, then the value functions defined by the iteration (3) admit

$$
H_{n}(t, w, i)=\frac{w^{\gamma}}{\gamma} \cdot a_{n}(t, i), \quad \forall(t, w, i) \in[0, T] \times[0,+\infty[\times \mathbb{D} \text { and } \forall n \in \mathbb{N},
$$

where the sequence of the functions of $\left\{a_{n}(t, i)\right\}_{n=0}^{\infty}$ is defined as follows.

$$
\begin{aligned}
a_{0}(t, i) & :=\left\langle\exp \left[\left(\mathbf{Q}^{T}+\gamma \mathbf{R}\right)(T-t)\right] \cdot \mathbf{e}_{i}, \mathbf{1}\right\rangle, \\
\mathbf{R} & :=\operatorname{diag}\left(r_{1}, r_{2} \ldots, r_{d}\right) \in \mathbb{R}^{d \times d} \\
\mathbf{1} & :=(1,1, \cdots, 1)^{T r} \in \mathbb{R}^{d}
\end{aligned}
$$

and

$$
a_{n+1}(t, i)=\mathscr{N} a_{n}(t, i), \quad \forall n \in \mathbb{N} .
$$

Here, $\mathscr{N}$ is a functional operator, and, for function $a(t, i)$ on $[0, T] \times \mathbb{D}$, it is defined as

$$
\mathscr{N} a(t, i):=e^{-\left(\lambda_{i}+q_{i}\right)(T-t)}+\int_{t}^{T} e^{-\left(\lambda_{i}+q_{i}\right)(s-t)} \sum_{j \neq i} q_{i j} a(s, j) d s,
$$


where

$$
\lambda_{i}:=\frac{1}{2}\left(\frac{\mu_{i}-r_{i}}{\sigma_{i}}\right)^{2} \frac{\gamma}{\gamma-1}-r_{i} \gamma
$$

If the agent has a CRRA utility $(\gamma=0)$ of

$$
U(x)=\ln x, \quad \text { for } x>0,
$$

then, for value functions $\left\{H_{n}(t, w, i)\right\}_{n=0}^{\infty}$, we have

$$
H_{n}(t, w, i)=\ln w+b_{n}(t, i), \quad \forall(t, w, i) \in[0, T] \times[0,+\infty[\times \mathbb{D} \text { and } \forall n \in \mathbb{N},
$$

where the sequence of the functions of $\left\{b_{n}(t, i)\right\}_{n=0}^{\infty}$ is defined as follows

$$
b_{0}(t, i):=\left.\frac{\partial a_{0}(t, i)}{\partial \gamma}\right|_{\gamma=0}=\left\langle\exp \left[\mathbf{Q}^{T}(T-t)\right] \cdot \mathbf{R}(T-t) \cdot \mathbf{e}_{i}, \mathbf{1}\right\rangle,
$$

and

$$
b_{n+1}(t, i)=\widetilde{\mathscr{N}} b_{n}(t, i), \quad \forall n \in \mathbb{N} .
$$

Here, $\widetilde{\mathscr{N}}$ is also a functional operator, and, for function $b(t, i)$ on $[0, T] \times \mathbb{D}$, it is defined as

$$
\widetilde{\mathscr{N} b}(t, i):=\int_{t}^{T} e^{-q_{i}(s-t)}\left(\widetilde{\lambda}_{i}+\sum_{j \neq i} q_{i j} b(s, j)\right) d s,
$$

where

$$
\tilde{\lambda}_{i}:=r_{i}+\frac{1}{2}\left(\frac{\mu_{i}-r_{i}}{\sigma_{i}}\right)^{2}
$$

Proof: First, for the utility function of

$$
U(x)=\frac{x^{\gamma}}{\gamma}, \quad \text { for } x \geq 0,
$$

where $\gamma<1$ and $\gamma \neq 0$, according to (2), we have

$$
\begin{aligned}
H_{0}(t, w, i) & =\frac{w^{\gamma}}{\gamma} \mathrm{E}\left[\exp \left(\gamma \int_{t}^{T}\langle\mathbf{r}, \boldsymbol{\alpha}(s)\rangle d s\right) \mid \boldsymbol{\alpha}(t)=\mathbf{e}_{i}\right] \\
& =\frac{w^{\gamma}}{\gamma} a_{0}(t, i),
\end{aligned}
$$

where the second equality follows from the characteristic function of the occupation times in the regime-switching model, which has been derived in Buffington and Elliott (2002). We conjecture that

$$
H_{1}(t, w, i)=\mathscr{M} H_{0}(t, w, i)=\frac{w^{\gamma}}{\gamma} g(t, i), \quad \forall(t, w, i) \in[0, T] \times[0,+\infty[\times \mathbb{D},
$$

where $g$ is a function on $[0, T] \times \mathbb{D}$. Because $\mathbf{H}_{1}=\mathscr{M} \mathbf{H}_{0}$ must satisfy the PDE of (21), with $\mathbf{v}$ replaced by $\mathbf{H}_{0}$, by substitution we can derive the following ordinary differential equation (ODE), that is satisfied by $g(\cdot, i)$ on $[0, T]$ for each fixed $i \in \mathbb{D}$.

$$
\begin{aligned}
& g_{t}(t, i)=\left(\lambda_{i}+q_{i}\right) g(t, i)-\sum_{j \neq i} q_{i j} a_{0}(t, j), \\
& g(T, i)=1,
\end{aligned}
$$


which yields

$$
g(t, i)=\mathscr{N} a_{0}(t, i)
$$

and

$$
H_{1}(t, w, i)=\mathscr{M} H_{0}(t, w, i)=\frac{w^{\gamma}}{\gamma} \mathscr{N} a_{0}(t, i)=\frac{w^{\gamma}}{\gamma} a_{1}(t, i)
$$

Then, by the same idea, we can conclude that if

$$
H_{n-1}(t, w, i)=\frac{w^{\gamma}}{\gamma} a_{n-1}(t, i)
$$

for an $n \geq 2$, then

$$
H_{n}(t, w, i)=\mathscr{M} H_{n-1}(t, w, i)=\frac{w^{\gamma}}{\gamma} \mathscr{N} a_{n-1}(t, i)=\frac{w^{\gamma}}{\gamma} a_{n}(t, i) .
$$

Second, for the utility function $(\gamma=0)$ of

$$
U(x)=\ln x, \quad \text { for } x \geq 0,
$$

according to (2), we have

$$
\begin{aligned}
H_{0}(t, w, i) & =\ln w+\mathrm{E}\left[\int_{t}^{T}\langle\mathbf{r}, \boldsymbol{\alpha}(s)\rangle d s \mid \boldsymbol{\alpha}(t)=\mathbf{e}_{i}\right] \\
& =\ln w+\left.\frac{\partial a_{0}(t, i)}{\partial \gamma}\right|_{\gamma=0} \\
& =\ln w+b_{0}(t, i) .
\end{aligned}
$$

We conjecture that

$$
H_{1}(t, w, i)=\mathscr{M} H_{0}(t, w, i)=\ln w+\widetilde{g}(t, i), \quad \forall(t, w, i) \in[0, T] \times[0,+\infty[\times \mathbb{D},
$$

where $\widetilde{g}$ represents a function on $[0, T] \times \mathbb{D}$. Considering (21) with $\mathbf{v}$ replaced by $\mathbf{H}_{0}$ in a similar way, we can derive the following $\operatorname{ODE}$ for $\widetilde{g}(\cdot, i)$ on $[0, T]$ for each fixed $i \in \mathbb{D}$.

$$
\begin{aligned}
& \widetilde{g}_{t}(t, i)=q_{i} \widetilde{g}(t, i)-\widetilde{\lambda}_{i}-\sum_{j \neq i} q_{i j} b_{0}(t, j), \\
& \widetilde{g}(T, i)=0,
\end{aligned}
$$

which yields

$$
\widetilde{g}(t, i)=\widetilde{\mathscr{N}} b_{0}(t, i)
$$

and

$$
H_{1}(t, w, i)=\mathscr{M} H_{0}(t, w, i)=\ln w+\widetilde{\mathscr{N}} b_{0}(t, i)=\ln w+b_{1}(t, i)
$$

Then, by the same idea, we can conclude that

$$
H_{n}(t, w, i)=\ln w+b_{n}(t, i), \quad \forall n \in \mathbb{N} .
$$

The proof is completed. 


\subsection{Approximate Solution to the Original Problem}

From the explicit form of (23), we can use the sequence of the value functions $\left\{\mathbf{H}_{n}\right\}$ to approximate the optimal value function $\mathbf{V}$ in the original problem. For a desirable level of accuracy, $\varepsilon>0$, iteration (3) can be carried out in the following way.

- Step 1: Set $n=0$ and $H_{0}(t, w, i)=\frac{w^{\gamma}}{\gamma} \cdot a_{0}(t, i)$, where

$$
a_{0}(t, i):=\left\langle\exp \left[\left(\mathbf{Q}^{T}+\gamma \mathbf{R}\right)(T-t)\right] \cdot \mathbf{e}_{i}, \mathbf{1}\right\rangle .
$$

- Step 2: Let $n=n+1$, obtain $a_{n}(t, i)$ through (24) and set

$$
H_{n}(t, w, i)=\frac{w^{\gamma}}{\gamma} \cdot a_{n}(t, i)
$$

- Step 3: Stop when

$$
\sup _{t, w, i}\left|H_{n}(t, w, i)-H_{n-1}(t, w, i)\right| \leq \varepsilon
$$

otherwise, return to Step 2.

Here, we consider only the case of $\gamma<1$ and $\gamma \neq 0$, although the case of $\gamma=0$ can be considered in a similar way.

\section{Exact Solution}

\subsection{Optimal Value Function}

Recall that optimal value function $\mathbf{V}$ satisfies the dynamic programming problem

$$
\mathbf{V}=\mathscr{M} \mathbf{V}
$$

Then, using (21), we can obtain a PDE for $\mathbf{V}$ and solve this PDE for the CRRA utility.

Proposition 5.1 For each fixed $i \in \mathbb{D}$, the optimal value function of $V(\cdot, \cdot, i)$ satisfies

$$
\frac{1}{2}\left(\mu_{i}-r_{i}\right)^{2} V_{W}^{2}(t, w, i)=\sigma_{i}^{2}\left[V_{t}(t, w, i)+r_{i} w V_{W}(t, w, i)+\sum_{j=1}^{d} q_{i j} V(t, w, j)\right] V_{W W}(t, w, i)
$$

on $[0, T[\times] 0,+\infty[$ and has boundary condition

$$
V(T, w, i)=\left\{\begin{array}{cl}
\frac{w^{\gamma}}{\gamma}, & \gamma<1, \gamma \neq 0 \\
\ln w, & \gamma=0
\end{array}\right.
$$


When $\gamma<1$ and $\gamma \neq 0$, this PDE has a solution given by

$$
V(t, w, i)=a(t, i) \frac{w^{\gamma}}{\gamma}, \quad \forall(t, w, i) \in[0, T] \times[0,+\infty[\times \mathbb{D},
$$

where $a(t, i)$ is the $i$-th component of an $\mathbb{R}^{d}$-valued column of

$$
\mathbf{a}(t):=(a(t, 1), a(t, 2), \cdots, a(t, d))^{T r} .
$$

It can also be shown that

$$
\mathbf{a}(t)=\exp [-(\boldsymbol{\Lambda}-\mathbf{Q})(T-t)] \cdot \mathbf{1},
$$

with

$$
\begin{aligned}
\Lambda & :=\operatorname{diag}(\lambda(1), \lambda(2), \cdots \lambda(d)) \in \mathbb{R}^{d \times d}, \\
\lambda(i) & :=\frac{1}{2}\left(\frac{\mu_{i}-r_{i}}{\sigma_{i}}\right)^{2} \frac{\gamma}{\gamma-1}-r_{i} \gamma .
\end{aligned}
$$

When $\gamma=0$, this PDE has a solution given by

$$
V(t, w, i)=\widetilde{a}(t, i) \ln w+g(t, i), \quad \forall(t, w, i) \in[0, T] \times[0,+\infty[\times \mathbb{D},
$$

where $\widetilde{a}(t, i)$ is the $i$-th component of an $\mathbb{R}^{d}$-valued column of

$$
\widetilde{\mathbf{a}}(t)=\exp (\mathbf{Q}(T-t)) \cdot \mathbf{1},
$$

and $g(t, i)$ is the $i$-th component of an $\mathbb{R}^{d}$-valued column of

$$
\mathbf{g}(t)=\exp (-\mathbf{Q} t) \cdot \int_{t}^{T} \exp (\mathbf{Q} s) \mathbf{b}(s) d s
$$

with

$$
\begin{aligned}
\mathbf{b}(t) & :=(b(t, 1), b(t, 2), \cdots, b(t, d))^{T r} \\
b(t, i) & :=\frac{1}{2}\left(\frac{\mu_{i}-r_{i}}{\sigma_{i}}\right)^{2} \widetilde{a}(t, i)+r_{i} \widetilde{a}(t, i) .
\end{aligned}
$$

Proof: By replacing $\mathbf{v}$ by $\mathbf{V}$ in (21), we can obtain a PDE for $\mathscr{M} V(\cdot, \cdot, i)$. Then, (27) follows from $\mathscr{M} V(\cdot, \cdot, i)=V(\cdot, \cdot, i)$, and the boundary condition holds trivially. To solve this PDE, we also consider the two following cases for the value of $\gamma$.

First, for the utility function of

$$
U(x)=\frac{x^{\gamma}}{\gamma}, \quad \text { for } x \geq 0,
$$

where $\gamma<1$ or $\gamma \neq 0$, we conjecture that

$$
V(t, w, i)=a(t, i) \frac{w^{\gamma}}{\gamma}
$$


where $a(\cdot, i)$ is a continuous function on $[0, T]$, with $a(T, i)=1$ for each $i \in \mathbb{D}$. It then follows that

$$
\begin{aligned}
V_{t}(t, w, i) & =a_{t}(t, i) \frac{w^{\gamma}}{\gamma}, \\
V_{W}(t, w, i) & =a(t, i) w^{\gamma-1}, \\
V_{W W}(t, w, i) & =a(t, i)(\gamma-1) w^{\gamma-2} .
\end{aligned}
$$

Substituting the foregoing into (27), for each $i \in \mathbb{D}$, we have

$$
a_{t}(t, i)=\left[\frac{1}{2}\left(\frac{\mu_{i}-r_{i}}{\sigma_{i}}\right)^{2} \frac{\gamma}{\gamma-1}-r_{i} \gamma\right] a(t, i)-\sum_{j=1}^{d} q_{i j} a(t, j),
$$

where the subscript of $t$ represents the derivative of $a(\cdot, i)$ with respect to $t$. We can also rewrite it in compact form as

$$
\mathbf{a}_{t}(t)=(\Lambda-\mathbf{Q}) \mathbf{a}(t)
$$

where

$$
\left.\mathbf{a}_{t}(t)=\left(a_{t}(t, 1), a_{t}(t, 2), \cdots a_{t}(t, d)\right)\right)^{T r}
$$

Then, (28) follows.

Second, for the utility function of

$$
U(x)=\ln x, \quad \text { for } x \geq 0,
$$

we conjecture that

$$
V(t, w, i)=\widetilde{a}(t, i) \ln w+g(t, i),
$$

where $\widetilde{a}(\cdot, i)$ and $g(\cdot, i)$ are both continuous functions on $[0, T]$, with $\widetilde{a}(T, i)=1$ and $g(T, i)=0$ for each $i \in \mathbb{D}$. It then follows that

$$
\begin{aligned}
V_{t}(t, w, i) & =\widetilde{a}_{t}(t, i) \ln w+g_{t}(t, i), \\
V_{W}(t, w, i) & =\widetilde{a}(t, i) \frac{1}{w}, \\
V_{W W}(t, w, i) & =-\widetilde{a}(t, i) \frac{1}{w^{2}} .
\end{aligned}
$$

Substituting the foregoing into (27), for each $i \in \mathbb{D}$, we have

$$
\begin{aligned}
& \widetilde{a}_{t}(t, i)=-\sum_{j=1}^{d} q_{i j} \widetilde{a}(t, j) \\
& g_{t}(t, i)=-\sum_{j=1}^{d} q_{i j} g(t, j)-\frac{1}{2}\left(\frac{\mu_{i}-r_{i}}{\sigma_{i}}\right)^{2} \widetilde{a}(t, i)-r_{i} \widetilde{a}(t, i),
\end{aligned}
$$

and, in compact form,

$$
\begin{aligned}
& \widetilde{\mathbf{a}}_{t}(t)=-\mathbf{Q} \widetilde{\mathbf{a}}(t), \\
& \mathbf{g}_{t}(t)=-\mathbf{Q g}(t)-\mathbf{b}(t),
\end{aligned}
$$

from which (29) and (30) follow. 
Remark 5.1 It is easy to see that we can not get a closed form solution to the optimal value function for a general utility function. However, since the characteristic function of the occupation times of the Markov chain is known (see Buffington and Elliott (2002)), $H_{0}$ defined by (2) can always be calculated (numerically). Then the iteration (3) provides a numerical method to solve the PDE (27) together with the initial point $H_{0}$.

\subsection{Optimal Portfolio Strategy}

In addition to the optimal value function, our original problem also requires us to specify an optimal portfolio process. Here we show that, even in a multiple-regime market, given any current state $i \in \mathbb{D}$, it remains optimal to invest our wealth in the same way as that given by the auxiliary problem in single regime $i$.

Proposition 5.2 For the dynamic programming problem in a single-regime market of $i \in \mathbb{D}$

$$
\mathscr{M} V(t, w, i)=\sup _{\pi \in \Pi_{t}} \mathrm{E}_{t, w, i}\left[1_{\left\{\xi_{t, 1}<T\right\}} V\left(\xi_{t, 1}, W_{\pi}\left(\xi_{t, 1}\right), \boldsymbol{\alpha}\left(\xi_{t, 1}\right)\right)+1_{\left\{\xi_{t, 1} \geq T\right\}} U\left(W_{\pi}(T)\right)\right],
$$

let $\hat{\pi}^{(i)}$ denote an optimal strategy, which can maximize the expectation on the right-hand side. Then, the optimal strategy for our original problem can be given by

$$
\langle\boldsymbol{\alpha}(t), \hat{\boldsymbol{\pi}}(t)\rangle, \quad \forall t \in[0, T],
$$

where $\hat{\boldsymbol{\pi}}(t)=\left(\hat{\pi}^{(1)}(t), \hat{\pi}^{(2)}(t), \cdots, \hat{\pi}^{(d)}(t)\right)^{T r}$.

Proof: Let $\hat{\pi}$ denote an optimal strategy for our original problem. Then for a fixed $i \in \mathbb{D}$ and $t \in[0, T]$, we can define strategy $\tilde{\pi} \in \Pi_{t}$ over $[t, T]$ as

$$
\tilde{\pi}(s)= \begin{cases}\hat{\pi}^{(i)}(s), & t \leq s \leq \xi_{t, 1} \wedge T \\ \hat{\pi}(s), & \xi_{t, 1} \wedge T<s \leq T\end{cases}
$$

and then we have

$$
\begin{aligned}
\mathscr{M} V(t, w, i) & =\mathrm{E}_{t, w, i}\left[1_{\left\{\xi_{t, 1}<T\right\}} V\left(\xi_{t, 1}, W_{\hat{\pi}^{(i)}}\left(\xi_{t, 1}\right), \boldsymbol{\alpha}\left(\xi_{t, 1}\right)\right)+1_{\left\{\xi_{t, 1} \geq T\right\}} U\left(W_{\hat{\pi}^{(i)}}(T)\right)\right] \\
& =\mathrm{E}_{t, w, i}\left[1_{\left\{\xi_{t, 1}<T\right\}} \mathrm{E}_{\xi_{t, 1}, W_{\hat{\pi}^{(i)}}\left(\xi_{t, 1}\right), \boldsymbol{\alpha}\left(\xi_{t, 1}\right)}\left[U\left(W_{\hat{\pi}}(T)\right)\right]+1_{\left\{\xi_{t, 1} \geq T\right\}} U\left(W_{\hat{\pi}^{(i)}}(T)\right)\right] \\
& =\mathrm{E}_{t, w, i}\left[U\left(W_{\tilde{\pi}}(T)\right)\right],
\end{aligned}
$$

where, considering the meaning of $\hat{\pi}$, for the second equality we can use

$$
V(t, w, i)=\mathrm{E}_{t, w, i}\left[U\left(W_{\hat{\pi}}(T)\right)\right], \quad \forall(t, w, i) \in[0, T] \times[0,+\infty[\times \mathbb{D} .
$$

Furthermore, it follows from $V(t, w, i)=\mathscr{M} V(t, w, i)$ that

$$
V(t, w, i)=\mathrm{E}_{t, w, i}\left[U\left(W_{\tilde{\pi}}(T)\right)\right],
$$

which is equivalent to saying that the strategy of $\tilde{\pi}$ is also an optimal strategy for our original problem. Therefore, at any point in time $t \in[0, T]$, given that $\boldsymbol{\alpha}(t)=\mathbf{e}_{i}$, to maximize the 
expected utility of the terminal wealth, it is optimal to follow the strategy $\hat{\pi}^{(i)}=\left(\hat{\pi}_{S}^{(i)}, \hat{\pi}_{O}^{(i)}\right)$, which is the optimal solution to the problem in a single-regime market of $i \in \mathbb{D}$.

From this proposition, by replacing the $\mathbf{M}$ by $\mathbf{V}$ in (22), we can show that the optimal strategy for our original problem in case of $\gamma<1$ and $\gamma \neq 0$, provided that the current state is $i$, satisfies

$$
\hat{\pi}_{O}^{(i)} \varepsilon_{O}^{(i)}+\hat{\pi}_{S}^{(i)}=-\frac{\left(\mu_{i}-r_{i}\right) V_{W}(t, w, i)}{\sigma_{i}^{2} w V_{W W}(t, w, i)}=\frac{\mu_{i}-r_{i}}{(1-\gamma) \sigma_{i}^{2}}
$$

\subsection{Further Analysis of the Optimal Portfolio}

At any point in time, the optimal portfolio in case of $\gamma<1$ and $\gamma \neq 0$ satisfies

$$
\begin{aligned}
\hat{\pi}_{O} \varepsilon_{O}+\hat{\pi}_{S} & =\frac{\mu-r}{(1-\gamma) \sigma^{2}}, \\
\hat{\pi}_{O}+\hat{\pi}_{S}+\hat{\pi}_{M} & =1
\end{aligned}
$$

where we suppress the superscript of $i$.

If we do not allow the risk-free bond in the optimal portfolio, i.e.,

$$
\hat{\pi}_{O}+\hat{\pi}_{S}=1
$$

then this implies that

$$
\begin{aligned}
\hat{\pi}_{S} & =\frac{\mu-r}{(1-\gamma) \sigma^{2}} \cdot \frac{1}{1-\varepsilon_{O}}-\frac{\varepsilon_{O}}{1-\varepsilon_{O}}, \\
\hat{\pi}_{O} & =-\frac{\mu-r}{(1-\gamma) \sigma^{2}} \cdot \frac{1}{1-\varepsilon_{O}}+\frac{1}{1-\varepsilon_{O}} .
\end{aligned}
$$

In Merton's well-known reduced optimization problem (see Merton $(1969,1971)$ ), which considers a portfolio of a stock and a risk-free bond, $\frac{\mu-r}{(1-\gamma) \sigma^{2}}$ is exactly the optimal percentage of wealth invested in the stock for CRRA utility. Therefore, we call the first term in our solution,

$$
\frac{\mu-r}{(1-\gamma) \sigma^{2}} \cdot \frac{1}{1-\varepsilon_{O}},
$$

the modified term of speculation. We call the second element,

$$
-\frac{\varepsilon_{O}}{1-\varepsilon_{O}},
$$

the term of delta hedging, because it is the solution of the delta hedging strategy. In fact, to render a delta-neutral portfolio containing a stock and an option, we need only set the portfolio's elasticity with respect to the stock to be equal to zero, i.e.,

$$
\varepsilon=\pi_{S}+\varepsilon_{O} \pi_{O}=0,
$$

which, combined with $\pi_{S}+\pi_{O}=1$, results in

$$
\pi_{S}=-\frac{\varepsilon_{O}}{1-\varepsilon_{O}} .
$$


If investment in a risk-free bond is permitted, then the optimal portfolio process cannot be determined uniquely, and we have only

$$
\hat{\pi}_{S}=\frac{\mu-r}{(1-\gamma) \sigma^{2}}-\hat{\pi}_{O} \varepsilon_{O}
$$

In contrast to the situation without a risk-free bond, the first term is a pure rather than modified term of speculation, and, according to (32), the second element is still called the term of delta hedging.

Therefore, for a portfolio comprising an option, an underlying stock and a risk-free bond, the allocation of wealth in the stock has the dual purpose of speculating for profits and hedging the risks involved in the option. More specifically, the smaller the $\gamma$, the less wealth will be invested in the stock for speculation. This is consistent with the Arrow-Pratt index of risk aversion for the CRRA utility

$$
-x U^{\prime \prime}(x) / U^{\prime}(x)=1-\gamma,
$$

which implies that the smaller the $\gamma$, the more risk averse the investor will be. And as $\gamma$ decreases, the investor becomes more risk averse, and the optimal investment strategy changes gradually from a speculative strategy to a delta hedging strategy.

\section{Conclusion}

An optimal asset allocation is studied in a regime-switching market consisting of an option, an underlying stock and a risk-free bond. It is true that if the market is complete, the derivative can be replicated by the stock and bond, but since derivatives are available in the market, investors can choose to invest in derivative market (in some cases, investor may hold some derivatives, he/she can optimize his/her portfolio using the strategy in the paper.) In our paper, the market is incomplete, so the problem is more meaningful. We provided both an approximate and an exact solution to the original portfolio optimization problem for power and logarithmic utility functions.

Since the market is incomplete, there is difficulty to represent the return of an option using the returns of a stock and a risk-free bond, and there is no simple closed form formula for the elasticity of the option price with respect to the stock price. To overcome this, we introduced a functional operator by which we can obtain a sequence of value functions. We shown that the optimal value function can be given by the limit of this sequence, and the original problem can be reduced to an auxiliary optimization problem in a single-regime market where the elasticities can be calculated. Hence, we solve this auxiliary problem by the elasticity approach and provide an approximation to the optimal value function. Our results show that, even in our multipleregime market, it remains optimal to invest our wealth in the same way as in a single-regime market.

The optimal strategy obtained in this study is a mixture of Merton's reduced portfolio optimization result and the delta-hedging strategy. Without a risk-free bond in the portfolio, the optimal percentage of the total wealth invested in the underlying stock is the sum of a modified Merton (see Merton $(1969,1971)$ ) speculative strategy and the delta hedging strategy. In the presence of a risk-free bond, in contrast, the modified term becomes a purely speculative 
term. Hence, the optimal allocation of the wealth in the stock constitutes an appropriate balance between speculating for profits and hedging the risks involved in the option. As the investor becomes more risk averse, the optimal investment strategy changes gradually from a speculative strategy to a delta hedging strategy.

\section{Acknowledgements}

The authors thank the anonymous referees for their valuable comments and suggestions to improve on earlier versions of this paper. H. Yang would like to thank the Research Grants Council of the Hong Kong Special Administrative Region, China (project No. HKU 706611P).

\section{References}

Ang, A., Chen, J. (2002). Asymmetric correlations of equity portfolios. Journal of Financial Economics, 63, 443-494.

Bekaert, G., Hodrick, R. (1993). On biases in the measurement of foreign exchange risk premiums. Journal of International Money and Finance, 12, 115-138.

Black, F., Scholes, M. (1973) The pricing of options and corporate liabilities. The Journal of Political Economy, 81, 637-654.

Bollen, N., Gray, S., Whaley, R. (2000). Regime switching in foreign exchange rates: Evidence from currency option prices. Journal of Econometrics, 94, 239-276.

Buffington, J., Elliott, R. (2002). American options with regime switching. International Journal of Theoretical and Applied Finance, 5, 497-514.

Çanakoğlu, E., Özekici, S. (2012). HARA frontiers of optimal portfolios in stochastic markets. European Journal of Operational Research, 221, 129-137.

Çelikyurt, U., Özekici, S. (2007). Multiperiod portfolio optimization models in stochastic markets using the mean-variance approach. European Journal of Operational Research, $179,186-202$.

Cox, J., Huang, C. (1989). Optimal consumption and portfolio policies when asset prices follow a diffusion process. Journal of Economic Theory, 49, 33-83.

Cox, J., Huang, C. (1991). A variational problem arising in financial economics. Journal of Mathematical Economics, 20, 465-487.

Fleming, W.H., Soner, H.M. (2006) Controlled Markov Processes and Viscosity Solutions (2nd ed.). Springer, New York.

Garcia, R., Perron, P. (1996). An analysis of the real interest rate under regime shifts. The Review of Economics and Statistics, 78, 111-125. 
Gray, S. (1996). Modeling the conditional distribution of interest rates as a regime-switching process. Journal of Financial Economics, 42, 27-62.

Guidolin, M., Timmermann, A. (2007). Asset allocation under multivariate regime switching. Journal of Economic Dynamics and Control, 31, 3503-3544.

Hamilton, J. (1989). A new approach to the economic analysis of nonstationary time series and the business cycle. Econometrica, 57, 357-384.

Karatzas, I., Lehoczky, J., Shreve, S. (1987). Optimal portfolio and consumption decisions for a small investor on a finite horizon. SIAM Journal on Control and Optimization, 25, $1557-1586$.

Korn, R. (2008). Optimal portfolios: New variations of an old theme. Computational Management Science, 5, 289-304.

Kraft, H. (2003). Elasticity approach to portfolio optimization. Mathematical Methods of Operations Research, 58, 159-182.

Markowitz, H. (1952). Portfolio selection. Journal of Finance, 7, 77-91.

Merton, R. (1969). Lifetime portfolio selection under uncertainty: The continuous-time case. The Review of Economics and Statistics, 51, 247-257.

Merton, R. (1971). Optimum consumption and portfolio rules in a continuous-time model. Journal of Economic Theory, 3, 373-413. 